\title{
One-dimensional plasmons in ultrathin metallic silicide wires of finite width
}

\author{
Eddy P. Rugeramigabo, Christoph Tegenkamp, and Herbert Pfnür \\ Institut für Festkörperphysik, Leibniz Universität Hannover, 30167 Hannover, Germany \\ Takeshi Inaoka \\ Department of Physics and Earth Sciences, Faculty of Science, University of the Ryukyus, \\ 1 Senbaru, Nishihara, Okinawa 903-0213, Japan \\ Tadaaki Nagao \\ International Center for Materials Nanoarchitechtonics, National Institute for Materials Science, 1-1 Namiki, Tsukuba 305-0044, Japan
}

(Received 11 January 2010; published 2 April 2010)

\begin{abstract}
The acoustic dispersion of plasmons (PLs) in narrow $(4 \mathrm{~nm})$ and ultrathin (one unit cell) metallic $\mathrm{DySi}_{2}$ wires, grown by self-assembly on vicinal $\mathrm{Si}(100)-[011] 4^{\circ}$ turns out to be unidirectional. We observed the lowest intersubband PL as well as the acoustic PL. These PLs are specific for narrow metallic strips of finite width. Our experimental and theoretical analysis suggests that only one of two electron pockets in the surface Brillouin zone makes a substantial contribution to the PLs because the other pocket has a much smaller conductive character due to a strong admixture of electronic states with $d$ character.
\end{abstract}

DOI: 10.1103/PhysRevB.81.165407

PACS number(s): 73.20.At, 68.65.-k, 73.21.- b, 73.25.+i

\section{INTRODUCTION}

Surface plasmons have recently been proven to be a versatile and highly efficient tool in subwavelength optics, biophotonics, and possibly also in data storage. ${ }^{1}$ In thin films or nanowires with cross-sectional dimensions on the order of the Fermi wavelength low-dimensional plasmons are predicted to exist with the plasmon energy vanishing in the long-wavelength limit. Especially in one dimension (1D) the single-particle excitation picture is predicted to break down and is replaced by a plasmon picture with a dispersion similar to sound waves. ${ }^{2}$ For applications this means that their short plasmonic wavelengths allow extreme confinement of light, a very localized conversion between plasmons and light by near-field coupling and signal transport with small distortions due to the almost linear dispersion (see below).

This opens many perspectives for potential applications but first requires a detailed knowledge about plasmonic properties such as their dispersion, which depends not only on electron density but on many details of the band structure, as we will show. In fact, there is still a lack of detailed understanding about the specific contributions of low-dimensional electronic bands close to the Fermi energy, $E_{F}$, to plasmonic excitations, the role of electronic correlations, the cross over from two-dimensional (2D) to 1D metallic systems or the decay mechansims of plasmons. Particularly attractive in this context are studies at the borderline between 1D and 2D, which can be well realized by self-organized adsorbed submonolayers (MLs) on flat and regularly stepped Si surfaces. These systems have in common that surface states of the underlying substrate are modified and partially filled with electrons by the adsorbate. They remain, however, located in the bulk band gap. ${ }^{3-5} \mathrm{In}$ isotropic $2 \mathrm{D}$ systems, e.g., in the $\mathrm{Ag}$ $\sqrt{3} \times \sqrt{3}$ phase $^{3,4,6}$ or in $\mathrm{DySi}_{2}$ monolayers on $\mathrm{Si}(111),{ }^{7}$ the acoustic plasmonic dispersion can be well described by a $2 \mathrm{D}$ nearly free-electron theory with still small but noticeable exchange-correlation corrections when the momentum $q_{\|}$is in the range as the Fermi wave vector $k_{F}$.
The plasmons investigated here originate from 1D wires of finite width generated by self-organization on a regularly stepped $\mathrm{Si}(001)$ surface, similar to those wirelike structures formed by submonolayers of $\mathrm{In}$ on $\mathrm{Si}(111)$ and $\mathrm{Au}$ on $\mathrm{Si}(557),{ }^{5,8-10}$ with corresponding potential deviations from Fermi-liquid behavior. ${ }^{9,11,12}$ In the simplest case, the Si substrate acts as a simple dielectric medium in the energy range of interest here but it mediates the coupling to two (and three) dimensions. It is therefore crucial for structure and array formation, as also demonstrated below. This scenario is very different from the formation of $2 \mathrm{D}$ acoustic surface plasmons generated by partially filled surface states of bulk metals, ${ }^{13,14}$ where the response of the bulk metal electrons has to be taken into account.

Intrinsic crossover from 1D to $2 \mathrm{D}$ behavior (i.e., within a single wire) is illustrated by our investigations, presented here, of plasmons confined in well separated, noninteracting metallic $\mathrm{DySi}_{2}$ wires of finite width. It is different from crossover behavior seen by extrinsic coupling (between many wires) once the separation between wires becomes comparable to the inverse of the wire-parallel momentum $q_{\| \cdot} \cdot{ }^{9,10}$ We not only find an acousticlike dispersion only along the wires, confirming $1 \mathrm{D}$ behavior at long wavelengths. The finite width of the strips is directly evident from the first experimental observation of intersubband plasmon excitation, which appears due to electron confinement perpendicular to the wires. It is also evident from the quantitative fit of the plasmon dispersion, which is only possible by taking the finite strip width explicitly into account. ${ }^{15,16}$ Third, we directly demonstrate the inefficiency of electronic states with strong $d$ character to plasmon excitations, which still allows the use of a nearly free-electron-gas (NFEG) model to describe almost quantitatively plasmonic excitations in a rareearth silicide.

\section{EXPERIMENTAL}

The experiments were carried out in ultrahigh vacuum at a base pressure of $7 \times 10^{-9} \mathrm{~Pa}$. This chamber contained a 
special high-resolution electron-loss spectrometer. It comprises a monochromatized electron beam and an energy analyzer, realized by electrostatic cylindrical $127^{\circ}$ deflection, combined with an electrostatic scan unit of a low-energy electron-diffraction (LEED) system. This instrument thus allows simultaneous high-momentum $\left(q_{\|}\right)$and high-energy resolution. ${ }^{17}$ Typical operating parameters were $23 \mathrm{meV}$ energy resolution at a $q_{\|}$resolution of $1.3 \times 10^{-2} \AA^{-1}$ in the energy range between 20 and $70 \mathrm{eV}$.

A uniaxial arrangement of $\mathrm{DySi}_{2}$ wires was prepared on a vicinal $\mathrm{Si}(100)-[011] 4^{\circ}$ surface either by adsorption of Dy at $770 \mathrm{~K}$ or by adsorption at room temperature and annealing at $770 \mathrm{~K}$ for $20 \mathrm{~min}$, as described in Ref. 18. Both procedures yielded identical results. Due to the uniaxial lattice mismatch of $\mathrm{DySi}_{2}$ compared to the $\mathrm{Si}$ crystal structure, wires with extremely high aspect ratios grow by self assembly.

The Si sample was carefully outgassed at temperatures up to $800{ }^{\circ} \mathrm{C}$ for several hours and finally heated to $1150{ }^{\circ} \mathrm{C}$, followed by slow cooling to room temperature over several minutes. This procedure yielded a homogeneously stepped surface with an average terrace width of 10.5 lattice constants, $a$, as checked with LEED.

\section{RESULTS AND DISCUSSION}

Low Dy concentrations, i.e., concentrations below 0.2 ML, do not change the surface structure upon the silicidation process. This concentration turns out to be a critical coverage for refacetting, which occurs once this concentration is exceeded. After adsorption of Dy in excess of 0.2 ML and silicidation as just described, an additional periodicity with a terrace width of $7.5 a$ appeared (see Fig. 1).

As it turns out, this new periodicity, which is close to the $(7 \times 2)$ reconstruction seen on flat $\mathrm{Si}(100)$, remains stable upon further adsorption of Dy. Its intensity saturates already for Dy concentrations above 0.5 ML. Remarkably, not only the average inclination of the surface is conserved but also the orientation of both types of facets, as judged from the shifts of peak positions as a function of electron energy. This requires a corresponding change in average step height for the shorter terraces but this is only a side aspect in the present context. In any case, the refacetting causes the formation of shorter and wider terraces, which seem to be directly related to the formation of "thin" and "thick" wires. The wire width of the thin wires of Ref. 18 corresponds exactly to the terrace width of $7.5 a$, whereas the thick wires have a width around $4 \mathrm{~nm} .{ }^{18}$ Most likely these $\mathrm{DySi}_{2}$ wires have hexagonal structure with the $c$ axis of the unit cell oriented along the surface but perpendicular to the wire direction, as found for thicker nanostructures on this surface. ${ }^{19}$ For the submonolayer Dy range this means that Dy must go below the first Si layer with a local concentration around one monolayer. ${ }^{20}$

Plasmonic losses can be measured by electron energy-loss spectroscopy (EELS) only after the refacetting of the surface sets in. This is demonstrated as an example in Fig. 2. Here plasmonic losses around $340 \mathrm{meV}$ are shown for $q_{\|}$ $=0.065 \AA^{-1}$, which become visible in EELS for coverages above $0.2 \mathrm{ML}$. At this value of $q_{\|}$a clear separation of the
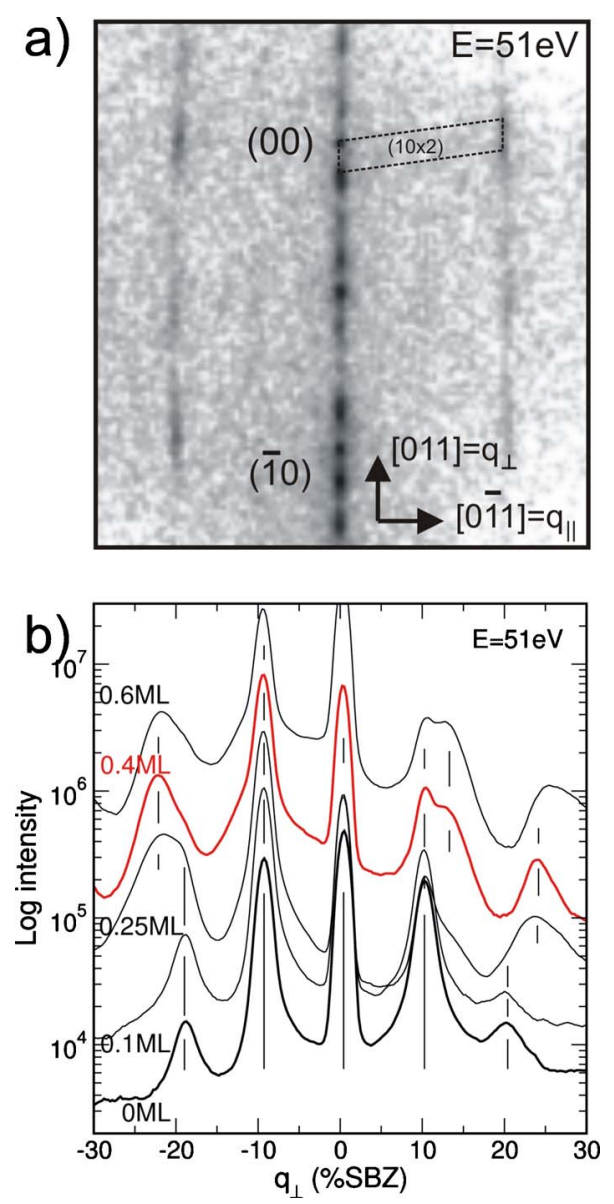

FIG. 1. (Color online) (a) LEED image of $0.5 \mathrm{ML}$ Dy on $\mathrm{Si}(001)-[011] 4^{\circ}$ after $\mathrm{DySi}_{2}$ formation. (b) LEED profiles taken along the $[0 \overline{1} \overline{1}]$ direction [see (a)] for different Dy coverages and a constant electron energy of $51 \mathrm{eV}$. The profiles are shifted against each other for better visibility. Peak shifts are due to Dy-induced refacetting.

loss peak from the Drude background and the elastic peak is possible. The loss-peak position is, within error bars, independent of the average $\mathrm{DySi}_{2}$ concentration, i.e., the underlying band structure (i.e., the density of states and the effective electron mass, $m^{*}$ ) is not changing upon further adsorption up to $0.5 \mathrm{ML}$. The full width at half maximum (FWHM) of the loss peak is around $250 \mathrm{meV}$ and thus an order of magnitude larger than the resolution of the instru-

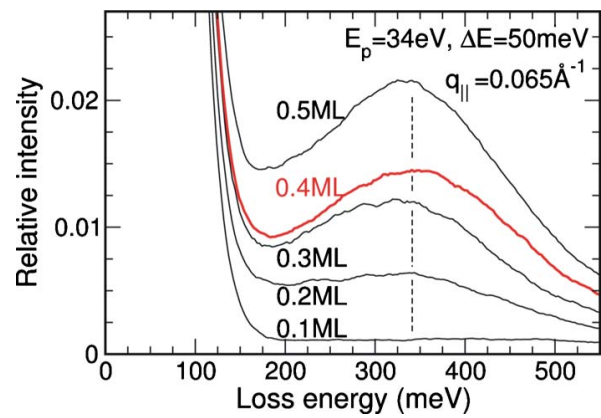

FIG. 2. (Color online) Electron energy-loss spectra taken at $q_{\|}=0.065 \AA^{-1}$ for different Dy coverages as indicated. 

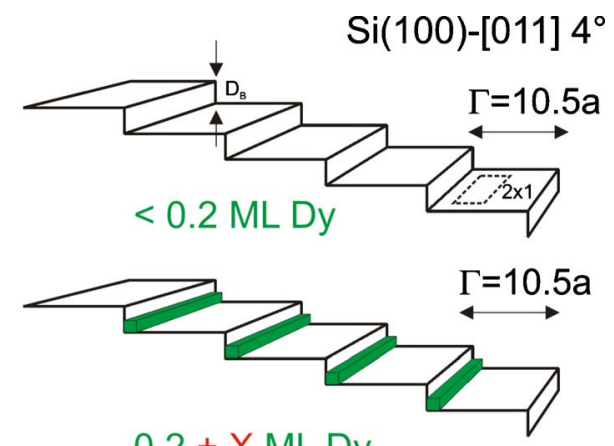

$0.2+X$ ML Dy

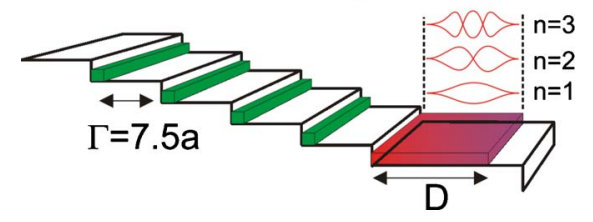

FIG. 3. (Color online) Model of narrow (dark, green bars) and wide $\mathrm{DySi}_{2}$ wires (dark, red sheet). Only the latter are metallic.

ment. This loss peak actually contains a substructure due to confinement of plasmons in a wire with finite width (see below).

These results are in agreement with most recent findings ${ }^{21}$ that the thin wires are semiconducting at room temperature. Therefore, these wires formed exclusively at low coverage do not contribute to plasmon formation. Only the wires with an average width close to $4 \mathrm{~nm}$, but of comparable height, formed at higher Dy coverages are metallic. As mentioned, the plasmon energy is insensitive to the concentration exceeding 0.2 ML. Therefore, we take a concentration of 0.42 of Dy as characteristic and concentrate on it throughout this paper. Only part of the concentration exceeding 0.2 ML is able to form metallic wires. These, according to the scanning tunnel microscope data of Ref. 18, are separated by more than $5 \mathrm{~nm}$ of nonmetallic interspace. Thus interaction between the metallic wires can be neglected. A schematic of this structure is shown in Fig. 3.

For this concentration, Fig. 4 shows EELS spectra as a function of in-plane momentum, $q_{\|}$along (left panel) and perpendicular to the wires (right panel). Only in the direction parallel to the wires, a dispersion up to $11 \%$ surface Brillouin zone $(\mathrm{SBZ})\left(q_{\|}=0.179 \AA^{-1}\right)$ is seen. In the perpendicular direction, the inelastic signal in generally very weak (please note the high magnification in the left part of Fig. 4), which is in sharp contrast to the high elastic diffuse background [see, e.g., Fig. 1(a)]. This is expected for a system that is not conducting in this direction. Only some phonon background (presumably mostly from the substrate) can be seen, together with a very weak peak around $300 \pm 50 \mathrm{meV}$, whose position is independent of the in-plane wave vector. This result with a one-dimensional dispersion corresponds well to the model of separated wires and is in qualitative agreement with that obtained for the electronic ground states with angular resolved photoemission spectroscopy (ARPES). ${ }^{18}$

Looking closer to these spectra, it is interesting to note that the half widths of the peaks is not continuously increasing as a function of $q_{\|}$. If we assume a single loss peak in the dispersion along the wires, we observe an extraordinary in-

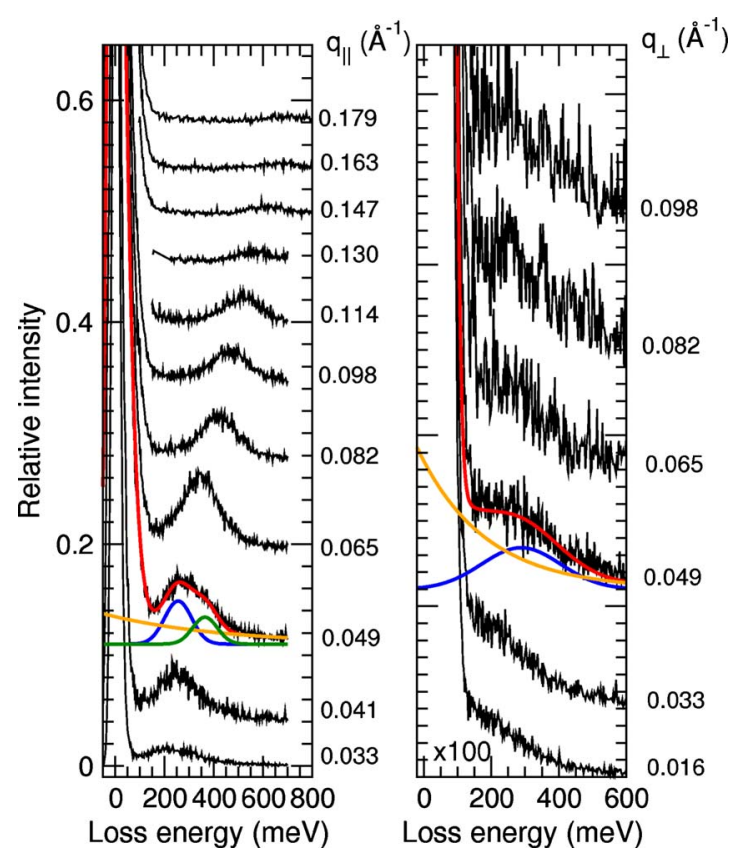

FIG. 4. (Color online) Loss spectra of $0.42 \mathrm{Dy} / \mathrm{Si}(100)-[011] 4^{\circ}$ along (left) and perpendicular(right) to the wire directions for different $q$ values $\left(E_{\text {prim }}=34 \mathrm{eV}\right)$. The accurate peak positions and the FWHM (cf. with Fig. 5) have been obtained from fitting the data including the elastic peak, an exponentially decaying Drude tail and two loss peaks. The contribution of these single peaks are shown exemplarily only for one spectrum in each direction. The resolution of the instrument was $30 \mathrm{meV}$. Please note the additional magnification in the right panel.

crease in the FWHMs again for small $q_{\|}$values (below $\left.0.125 \AA^{-1}\right)$. This is better visible in Fig. 5(b), where we plotted the FWHM for the plasmon losses along the wires. The assumption of a single plasmonic loss would result in an increase in the FWHMs at small plasmonic losses indicated by the dashed line (which marks the average FWHM obtained from different measurements at various electron energies), which is unphysical. It is a clear indication for a double-peak structure, which for some spectra is directly visible, e.g., at $q_{\|}=0.049 \AA^{-1}$ (see Fig. 4). This phenomenon appeared in all data sets taken at small $q_{\|}$and our fitting procedure therefore had to take into account two peaks in this $q$ range [see the compilation of characteristic losses in Fig. 5(a)].

This finding of a multiple plasmonic excitation should in fact be expected for the low-dimensional structure under investigation here, i.e., for metallic stripes of finite width. This geometry can be regarded as a two-dimensional system confined in one of these dimensions. This confinement results in a multiplet of quasi-one-dimensional plasmonic dispersions forming plasmonic subbands. This means that the doublet structure observed here corresponds to an intersubband transition of a low-dimensional plasmon. Indeed, if we take the simple particle-in-a-box model with infinite potential wells in the confined direction and the strip width of $L=40 \AA$ from above, we obtain an eigenenergy $E_{n}=n^{2} h^{2} / 8 m^{*} L^{2}$ $=47 n^{2} \mathrm{meV}(n=1,2,3, \ldots)$. Our calculations show that three subbands are occupied so that the lowest intersubband mode 


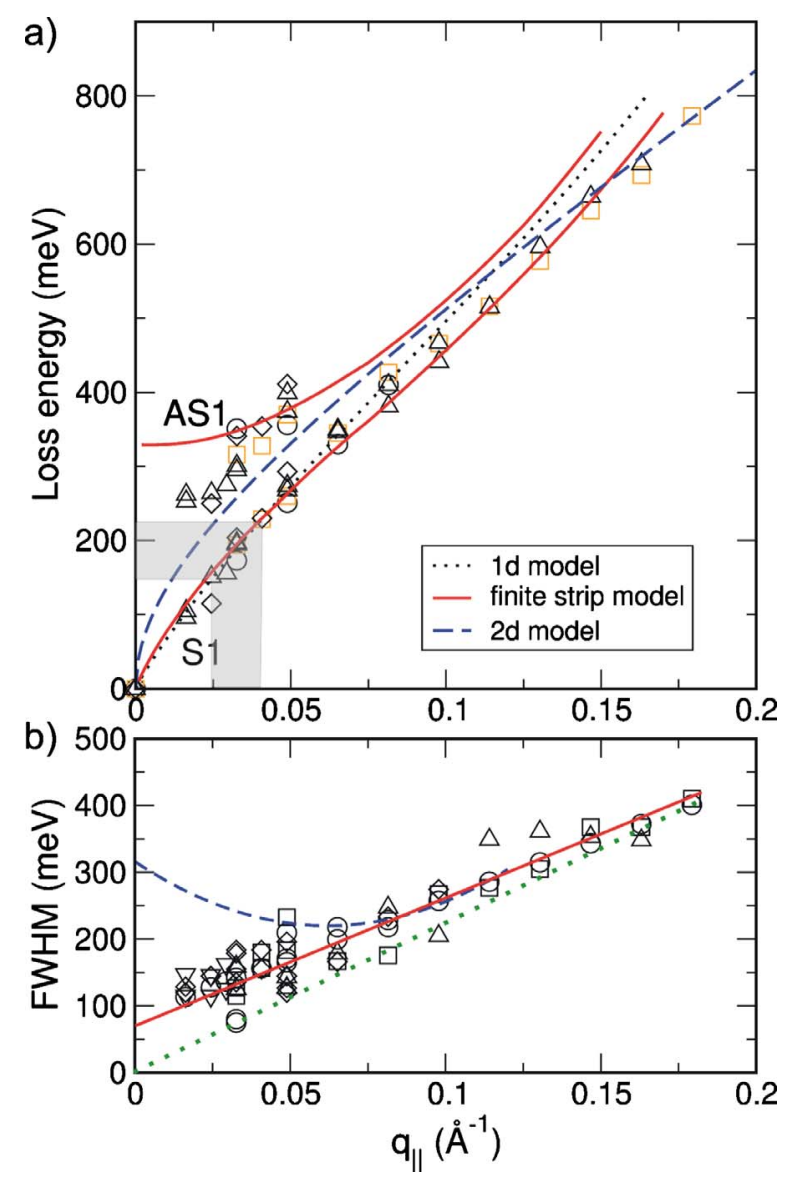

FIG. 5. (Color online) (a) Dispersion of the plasmon in $\mathrm{DySi}_{2}$ wires along the wire direction. Different symbols mark different data sets. Lines are fits with an effective mass of $0.5 m_{e}$ for a $2 \mathrm{D}$ model using the random-phase approximation (Ref. 22) $\left(N_{2 \mathrm{D}}=8\right.$ $\times 10^{13} \mathrm{~cm}^{-2}$, dashed), a $1 \mathrm{D}$ density of $N_{1 \mathrm{D}}=1.4 \times 10^{7} \mathrm{~cm}^{-1}$ (dots) on an $8 \AA$ wide chain using a local-field correction (dots), and full consideration of exchange and correlation on a finite $4 \mathrm{~nm}$ wide strip $\left(N_{1 \mathrm{D}}=3.2 \times 10^{7} \mathrm{~cm}^{-1}\right.$ or $N_{2 \mathrm{D}}=8 \times 10^{13} \mathrm{~cm}^{-2}$, lines $)$. (b) FWHM of the loss peaks. The dashed line shows the average of points is corrected by the finite the FWHM assuming only a single peak. The experimental data energy resolution of $30 \mathrm{meV}$. The green dotted curve is obtained after deconvolution of the finite integration width in momentum space. For more details see text.

has an energy of $E_{4}-E_{3}=329 \mathrm{meV}$, in reasonable agreement with the extrapolation of our measurements to $q_{\|}=0$. Here $h$ denotes the Planck's constant. The effective electron mass $m^{*}=0.5 m_{e}$ was taken from the average of the ARPES measurements. ${ }^{18}$ This excitation energy is also close to the dispersionless small loss peak found in [0 $\overline{1} \overline{1}]$ direction and is thus also consistent with this model.

In order to explore the plasmon dispersion and decay mechanisms in detail, the spectra have been fitted to obtain the peak position and the FWHM accurately. The results for various electron-beam energies are shown in Figs. 5(a) and 5(b). For the lowest plasmon mode, the extrapolation to zero excitation energy in the long-wavelength limit, characteristic for 1D and 2D plasmons, is fully compatible with our data. Due to the almost linear initial dispersion, the plasmon group velocity $v_{\mathrm{PL}}$ is almost independent of frequency, i.e., the plasmon will ideally not disperse. We obtain $v_{\mathrm{PL}} \approx 10^{6} \mathrm{~m} / \mathrm{s}$, which is two orders of magnitude higher than the Fermi velocity of the electrons originating from the states around $\bar{\Gamma}$.

For a quantitative comparison with theory, we refer to ARPES measurements carried out on the same system with a similar wire distribution. ${ }^{18}$ According to Ref. 18, a surface band centered at the $\bar{\Gamma}$ and a split band ${ }^{21}$ centered at $\bar{J}$ point cross the Fermi energy. The bands can be described by parabolas with effective masses of $0.45 m_{e}$ (band at $\bar{\Gamma}$ ) and $0.54 m_{e}$ (band at $\left.\bar{J}\right)$, respectively. From the Fermi wave vector $k_{F}$ the corresponding electron densities are estimated to be $6.5 \times 10^{13} \mathrm{~cm}^{-2}$ and $2.3 \times 10^{14} \mathrm{~cm}^{-2}$, respectively $\left(2 \pi N_{2 \mathrm{D}}\right.$ $\left.=k_{F}^{2}\right)$. They correspond to $1 \mathrm{D}$ densities of $1.3 \times 10^{7} \mathrm{~cm}^{-1}$ and $2.4 \times 10^{7} \mathrm{~cm}^{-1}$, respectively. Although, from the band structure just described, clear deviations from a NFEG description must be expected, our fits with an effective mass of $0.5 m_{e}$ show that there is still remarkably good qualitative agreement of the data with the NFEG model. However, this is true only if exchange and correlation effects and the finite width are taken into account properly. Therefore, no reasonable fit can be obtained when dispersion is calculated within the random-phase approximation for the $2 \mathrm{D}$ electron gas. ${ }^{22} \mathrm{It}$ systematically deviates from the data points irrespective of the $N_{2 \mathrm{D}} / m^{*}$ values [dashed line in Fig. 5(a)]. A much better fit is already obtained with a $1 \mathrm{D}$ density of $N_{1 \mathrm{D}}=1.4$ $\times 10^{7} \mathrm{~cm}^{-1}$ confined on an $8 \AA$ wide chain by a harmonicoscillator-type potential, including local-field corrections. The dispersion curve in this model, however, is too straight and does not reproduce the side band observed since it describes a stripe that is much narrower than those investigated here. Only the model by Inaoka ${ }^{16}$ that takes both the proper finite strip width and electron correlations into account (lines) reproduces quantitatively the characteristic dispersion throughout the whole $q_{\|}$range. It also reproduces the second branch with finite energy and near $q_{\|} \rightarrow 0$. The upper red curve is within error bars, still compatible with our data, and intersects with the ordinate at around $330 \mathrm{meV}$. It thus agrees reasonably well with the finite-energy mode found in [011] direction. Therefore, the assignment of this mode to the lowest intersubband plasmon, is consistent with the theoretical results by Inaoka. ${ }^{16}$ This lowest intersubband plasmon mode in a single strip of finite width has one node in its induced charge-density distribution perpendicular to the wire.

Although the agreement mentioned above is very satisfactory, the quantitative comparison with the electron densities at $E_{F}$ from experiment ${ }^{18}$ differs significantly from that derived from our fits. With an effective mass of 0.5 , consistent with ARPES, the electron density of $8 \times 10^{13} \mathrm{~cm}^{-2}$ derived from our best fit for the plasmon dispersion is about $20 \%$ higher than the density from ARPES at $E_{F}$ centered at $\bar{\Gamma}$ but about three times smaller than that centered at $\bar{J}$. The latter bands have a strong admixture of $d$ electrons, as obvious from the dispersions of the occupied electronic bands. ${ }^{18}$ Therefore, it seems that the semilocalized character of $d$ electrons, which is not taken into account in our model, reduces the contribution of the electron pocket around the $\bar{J}$ to the plasmonic excitations drastically. Interestingly, this effect can be taken into account just by renormalizing the electron density in the $q_{\|}$range investigated here. 
The FWHM of the plasmon losses as measured and shown by the data points of Fig. 5(b) turn out to be largely dominated not by the finite-energy resolution of $\Delta E_{\text {res }}$ $=30 \mathrm{meV}$ of the instrument, as judged from the FWHM of elastically scattered peak. Although we have used an instrument with very good $q$-space resolution, it is still governed by instrumental $q$-space broadening. This is illustrated by the gray bars in Fig. 5(a). As indicated there, the finite $q$-resolution results in an effective energy broadening for a dispersing plasmon. In order to get a consistency check with the $q$ resolution determined from the elastic peak, we extrapolated the average FWHM obtained for different experimental sets to $q_{\|} \rightarrow 0$, as shown by the red (solid) line in Fig. 5(b). It intersects the energy axis at around $70 \mathrm{meV}$ for $q_{\|}$ $\rightarrow 0$. Taking the minimum FWHM in $q$ space of the elastic (00) peak, which is close to $1 \% \mathrm{SBZ}$, it turns out that this residual energetic half width of the plasmon losses is completely due to this finite $q$-space resolution. Deconvoluting this contribution together with the finite energetic resolution, the green dotted line results, which now may be correlated with an inverse lifetime.
Again an almost linear increase in half widths is seen over the whole range of plasmon dispersion, which is characteristic for decay mechanisms involving many-body effects. For a more detailed analysis, quantitatively reliable band-structure calculations are needed but not available yet.

\section{CONCLUSIONS}

Higher excited plasmonic modes are expected and seen on the ultrathin and ultranarrow silicide wires investigated here that are separable from the lowest band of acoustic plasmons. Indeed, we were able to identify both this band and the first intersubband plasmon. Both are dispersing only along the wires, indicating weak coupling between wires. The experimentally determined one-dimensional dispersion can very well be described within the nearly free-electron-gas model, taking electron correlations and the finite width into account properly. The reason is that mainly $s p$ bands contribute to plasmonic excitations, whereas the $d$-state character of the more localized electrons in the $s p d$ band centered at the $\bar{J}$ point strongly reduces their contribution to the effective plasmonic electron density.
${ }^{1}$ D. E. Chang, A. S. Sørensen, P. R. Hemmer, and M. D. Lukin, Phys. Rev. Lett. 97, 053002 (2006).

${ }^{2}$ S. Tomonaga, Prog. Theor. Phys. 5, 544 (1950).

${ }^{3}$ T. Inaoka, T. Nagao, S. Hasegawa, T. Hildebrandt, and M. Henzler, Phys. Rev. B 66, 245320 (2002).

${ }^{4}$ T. Nagao, T. Hildebrandt, M. Henzler, and S. Hasegawa, Phys. Rev. Lett. 86, 5747 (2001).

5 J. N. Crain and F. J. Himpsel, Appl. Phys. A: Mater. Sci. Process. 82, 431 (2006).

${ }^{6}$ T. Nagao, T. Hildebrandt, M. Henzler, and S. Hasegawa, Surf. Sci. 493, 680 (2001).

${ }^{7}$ E. P. Rugeramigabo, T. Nagao, and H. Pfnür, Phys. Rev. B 78, 155402 (2008).

${ }^{8}$ J. R. Ahn, J. H. Byun, H. Koh, E. Rotenberg, S. D. Kevan, and H. W. Yeom, Phys. Rev. Lett. 93, 106401 (2004).

${ }^{9}$ T. Nagao, S. Yaginuma, T. Inaoka, and T. Sakurai, Phys. Rev. Lett. 97, 116802 (2006).

${ }^{10}$ C. Liu, T. Inaoka, S. Yaginuma, T. Nakayama, M. Aono, and T. Nagao, Phys. Rev. B 77, 205415 (2008).

${ }^{11}$ J. R. Ahn, H. W. Yeom, H. S. Yoon, and I.-W. Lyo, Phys. Rev. Lett. 91, 196403 (2003).
${ }^{12}$ D. Sánchez-Portal, S. Riikonen, and R. M. Martin, Phys. Rev. Lett. 93, 146803 (2004).

${ }^{13}$ B. Diaconescu, K. Pohl, L. Vattuone, L. Savio, Ph. Hofmann, V. M. Silkin, J. M. Pitarke, E. V. Chulkov, P. M. Echenique, D. Faraias, and M. Rocca, Nature (London) 448, 57 (2007).

${ }^{14}$ V. M. Silkin, J. M. Pitarke, E. V. Chulkov, B. Diaconescu, K. Pohl, L. Vattuone, L. Savio, Ph. Hofmann, D. Farias, M. Rocca, and P. M. Echenique, Phys. Status Solidi A 205, 1307 (2008).

${ }^{15}$ T. Inaoka, J. Phys. Soc. Jpn. 73, 2201 (2004).

${ }^{16}$ T. Inaoka, Phys. Rev. B 71, 115305 (2005).

${ }^{17}$ H. Claus, A. Büssenschütt, and M. Henzler, Rev. Sci. Instrum. 63, 2195 (1992).

${ }^{18}$ C. Preinesberger, G. Pruskil, S. K. Becker, M. Dähne, D. V. Vyalikh, S. L. Molodtsov, C. Laubschat, and F. Schiller, Appl. Phys. Lett. 87, 083107 (2005).

${ }^{19}$ G. F. Ye, M. A. Crimp, and J. Nogami, Phys. Rev. B 74, 033104 (2006).

${ }^{20}$ C. Bonet, D. J. Spence, and S. P. Tear, Surf. Sci. 504, 183 (2002).

${ }^{21} \mathrm{M}$. Wanke and M. Dähne (private communication).

${ }^{22}$ F. Stern, Phys. Rev. Lett. 18, 546 (1967). 